\title{
Saturación arterial de oxígeno y frecuencia cardíaca en recién nacidos de término sanos durante la primera hora post nacimiento*
}

\author{
Arterial oxygen saturation and heart rate in term newborn \\ in the first hour after birth
}

\author{
Aldo Bancalari ${ }^{\mathrm{a} b}$, Heriberto Araneda ${ }^{\mathrm{c}}$, Patricia Echeverria ${ }^{\mathrm{c}}$, \\ Andrés Marinovic ${ }^{\mathrm{a}}$, Camilo Manríquez ${ }^{\mathrm{c}}$
}

aDepartamento Pediatría, Facultad de Medicina, Universidad de Concepción

bServicio Neonatología, Hospital Guillermo Grant Benavente, Concepción

'Departamento Obstetricia y Ginecología, Facultad de Medicina, Universidad de Concepción

Recibido: 19 de noviembre de 2018; Aceptado: 28 de febrero de 2019

\begin{abstract}
Resumen
El oxímetro de pulso ha sido útil en la evaluación cardiorrespiratoria del neonato. Objetivo: Evaluar la saturación arterial de oxígeno $\left(\mathrm{SpO}_{2}\right)$ y frecuencia cardíaca (FC) en recién nacidos ( $\left.\mathrm{RN}\right)$ en los primeros 60 minutos después del nacimiento. Pacientes y Método: Estudio observacional prospectivo en RN de término sanos, nacidos por vía vaginal o cesárea, con apego materno, efectuado a nivel del mar. Se obtuvo registro continuo de $\mathrm{SpO}_{2}$ post-ductal y de FC desde el minuto 1 al 10 post-natal, y luego a los 15, 30 y 60 minutos. $\mathrm{La} \mathrm{SpO}_{2}$ y FC se midieron con un oxímetro de pulso Nellcor. Para el análisis estadístico se utilizó programa Stata v.14. Resultados: Se enrolaron 324 RNT sanos, que cumplieron criterios de inclusión, de los cuales 160 nacieron por vía vaginal y 164 por cesárea. $\mathrm{La} \mathrm{SpO}_{2}$ aumentó progresivamente desde el minuto 1 (58,7\%) hasta el minuto 10 (94,5\%). Los RN por vía vaginal presentaron una $\mathrm{SpO}_{2}$ significativamente mayor hasta el minuto 10 post-natal respecto a los nacidos por cesárea $(\mathrm{p}<0,001)$. En los neonatos nacidos por vía vaginal, la FC fue significativamente mayor en los 2 primeros minutos post-natales, y luego desde el minuto 10 al 60 ( $p<0,003$ ). No se encontraron diferencias por género en $\mathrm{SpO}_{2}$ y FC. Conclusión: En $\mathrm{RNT}$ la $\mathrm{SpO}_{2}$ post-natal aumenta progresivamente, siendo mayor en los primeros 10 minutos en los nacidos por vía vaginal. En los RN por vía vaginal se observó también una FC mayor en los primeros y últimos minutos evaluados.
\end{abstract}

Palabras clave: Saturación de oxígeno; oximetría de pulso; frecuencia cardiaca; recién nacido de término

*Artículo distinguido con el segundo lugar categoría general en el 58 Congreso Chileno de Pediatría, "Premios Dr. Alfredo Commentz L." efectuado del 3 al 6 de Octubre de 2018 en Antofagasta.

Correspondencia:

Aldo Bancalari Molina

aldobancalari@gmail.com 


\begin{abstract}
The pulse oximeter has been useful in the cardiorespiratory evaluation of the newborn. Objective: To assess arterial oxygen saturation $\left(\mathrm{SpO}_{2}\right)$ and heart rate (HR) in newborns in the first 60 minutes after birth. Patients and Method: Prospective observational study in healthy term newborns, delivered vaginally or by cesarean section, with maternal bonding, carried out at sea level. A continuous postductal $\mathrm{SpO}_{2}$ and $\mathrm{HR}$ record were obtained from minute 1 to 10 after birth, and then at 15, 30 and 60 minutes. The $\mathrm{SpO}_{2}$ and HR were measured with a Nellcor pulse oximeter. The software Stata v.14 was used for the statistical analysis. Results: 324 healthy term newborns that met the inclusion criteria were included, of which 160 born vaginally and 164 by cesarean section. The $\mathrm{SpO}_{2}$ increased progressively from minute $1(58.7 \%)$ to minute 10 (94.5\%). Newborns delivered vaginally had a significantly higher $\mathrm{SpO}_{2}$ until minute 10 after birth than those born by cesarean section $(\mathrm{p}<0.001)$. In newborns delivered vaginally, HR was significantly higher in the first two minutes after birth, and then from minute 10 to 60 ( $\mathrm{p}<0.003$ ). There were no differences by gender in $\mathrm{SpO}_{2}$ and HR. Conclusion: In term newborns, the $\mathrm{SpO}_{2}$ increases progressively, being higher in the first 10 minutes in those born vaginally. In newborns delivered vaginally, a higher HR was also observed in the first and last minutes evaluated.
\end{abstract}

Keywords:

Oxygen saturation; pulse oximetry; heart rate; term newborn

\section{Introducción}

En el recién nacido $(\mathrm{RN})$, la función de la placenta termina luego de que el cordón es ligado, dando inicio a un período transicional, en el cual la oxigenación, aumenta en forma progresiva durante los primeros minutos posterior al nacimiento, progresión que puede ser medida a través del oxímetro de pulso ${ }^{1}$. Los procesos fisiológicos que ocurren durante el período postnatal inmediato, especialmente respecto a saturación arterial de oxígeno $\left(\mathrm{SpO}_{2}\right)$ y frecuencia cardíaca (FC) han sido objeto de investigación en los últimos años².

En base a estudios de saturación de oxígeno en recién nacidos de término sanos en los primeros minutos de vida posterior al nacimiento ${ }^{2,5,7,8}$, la Academia Americana de Pediatría y la Academia Americana del Corazón (AAP-AHA) ${ }^{3}$ en el año 2010 determinaron los objetivos de $\mathrm{SpO}_{2}$ durante los primeros 10 minutos de vida post-natales, estableciendo de esta manera, la saturación arterial de oxígeno, que debe presentar un recién nacido en los primeros minutos de vida postnatales ${ }^{3}$. La tabla de los objetivos de $\mathrm{SpO}_{2}$ ha resultado fundamental para evaluar la efectividad de las maniobras de reanimación y para evitar una innecesaria administración de oxígeno en los primeros minutos postnatales en los recién nacidos sanos ${ }^{3}$.

La oximetría de pulso, es un procedimiento simple, indoloro, no invasivo, fácil de utilizar y de bajo costo, para la monitorización continua y objetiva de la saturación arterial de oxígeno y de la frecuencia cardiaca ${ }^{4}$. El saturómetro mide sensiblemente los cambios cardiopulmonares que ocurren durante la adaptación del recién nacido en el periodo post-natal y en otras situaciones clínicas, constituyéndose en una herramienta útil en la evaluación de los RN en sala de parto y en las Unidades Neonatales.
La transición cardiopulmonar del feto al $\mathrm{RN}$ es un proceso complejo que requiere de algunos minutos para lograr la estabilización ${ }^{6,7}$. Después del pinzamiento del cordón umbilical, se producen diversos cambios fisiológicos, produciéndose un aumento progresivo de la $\mathrm{SpO}_{2}$ en pocos minutos a valores definitivos ${ }^{7}$. Diversos factores pueden influir en la saturación de oxígeno en los primeros minutos post-natales, tales como; el lugar de nacimiento; que ocurra a nivel del mar o en altitud; según la vía de resolución del parto (vaginal o cesárea); o según el nivel de vitalidad del $\mathrm{RN}^{8-12}$.

El objetivo del presente estudio fue evaluar la saturación arterial de oxígeno y frecuencia cardiaca en recién nacidos de término en los primeros 60 min después del nacimiento.

\section{Pacientes y Método}

Estudio cuantitativo, observacional, cohorte prospectiva, en recién nacidos de término sanos, por vía vaginal o por cesárea, que no requirieron reanimación ni oxígeno adicional.

El estudio se realizó en la unidad de cuidados inmediatos neonatales del Hospital Guillermo Grant Benavente de Concepción (HGGB), entre julio y diciembre del año 2012. Este centro asistencial se encuentra a nivel del mar (presión atmosférica $760 \mathrm{mmHg}$ ).

Los neonatos incluidos fueron aquellos cuyas madres aceptaron participar en el estudio y en la medida que algún miembro del equipo investigador estaba disponible, para registrar la medición de $\mathrm{SpO}_{2}$ y FC. El equipo de investigación no participó en el cuidado

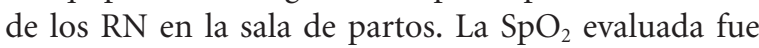
post-ductal para no interferir con el apego materno efectuado en pabellón. Todos los recién nacidos reci- 
bieron apego de acuerdo a protocolo establecido en nuestro hospital; 30 min para los nacidos por vía vaginal y 10 min para los nacidos por cesárea.

Se enrolaron $351 \mathrm{RN}$ de término sanos, de los cuales se excluyeron 27, por dificultades técnicas en obtener la señal de saturometría y frecuencia cardiaca antes de los 2 min postparto. La muestra final en estudio fue de $324 \mathrm{RN}$ quienes no requirieron ningún tipo de intervención y fueron seleccionados mediante muestreo no probabilístico por conveniencia.

Los criterios de inclusión en el estudio fueron: nacimiento único sano, con edad gestacional $\geq 37$ semanas, peso adecuado para la edad gestacional, sin evidencia de hipoxia ni antecedentes de morbilidad o complicación materna y con buena señal del oxímetro de pulso antes de los $2 \mathrm{~min}$ posteriores al pinzamiento del cordón umbilical.

Criterios de exclusión: se excluyeron todos los partos que terminaron en cesárea de urgencia; aquellos recién nacidos cuyo padre o madre no asintió participar del estudio y recién nacidos de madres que recibieron oxígeno, morfina y/o esteroides durante el trabajo de parto.

El pinzamiento del cordón umbilical fue en la mayoría de los RN después del primer llanto, lo cual ocurrió entre los 30 y 60 segundos posterior al nacimiento. Se colocó el sensor en la región anterior del pie derecho (arteria dorsal anterior), correspondiendo a $\mathrm{SpO}_{2}$ postductal, lo más pronto posible y se obtuvo un registro continuo de $\mathrm{SpO}_{2}$ y FC desde el minuto 1 al 10 después del parto. Posteriormente la $\mathrm{SpO}_{2}$ y la FC se registraron a los 15,30 y 60 min sin remover el oxímetro de pulso. Los tiempos se midieron con un cronómetro que se inició después de la ligadura del cordón umbilical. La $\mathrm{SpO}_{2}$ y la FC se midieron con un saturómetro Nellcor modelo OxiMax N-600 (Mansfield, MA, USA). La sonda se conectó al cable de oximetría antes de aplicarse al recién nacido, ya que aumenta la confiabilidad y la velocidad con la que se muestran los parámetros analizados ${ }^{13}$.

No se realizó aspiración oronasofaríngea de rutina en los RN según protocolo del Servicio de Neonatología de HGGB, de acuerdo a experiencia local y según lo publicado por Gungor y $\operatorname{cols}^{14}$. La puntuación de Apgar fue asignada por los pediatras o matronas de turno que atienden a los RN en el momento del parto.

En aquellas madres que se efectuó operación cesárea recibieron anestesia epidural, mientras que en los partos vaginales se utilizó sólo anestesia local.

La temperatura ambiente del pabellón y de la sala de cuidados inmediatos osciló entre $24^{\circ} \mathrm{C}$ y $26^{\circ} \mathrm{C}$ y los $\mathrm{RN}$ fueron recibidos en cuna radiante, con servo control de temperatura. La temperatura axilar de los $\mathrm{RN}$ osciló entre $36,5^{\circ} \mathrm{C}$ y $37^{\circ} \mathrm{C}$, durante el periodo estudiado.
Este estudio fue aprobado por el Comité Ético Científico del Servicio de Salud Concepción y se obtuvo el consentimiento informado de uno de los padres previo al parto.

Los investigadores que registraron la información de $\mathrm{SpO}_{2}$ y $\mathrm{FC}$ eran independientes del cuidado posterior del $\mathrm{RN}$.

\section{Análisis estadístico}

Para el almacenamiento de la información, se creó una base de datos en Microsoft Excel, para luego ser analizada en el programa estadístico Stata V. 14.

Se utilizó análisis univariado: para las variables cualitativas se calculó frecuencia absoluta y frecuencia relativa porcentual, mientras que para las variables cuantitativas se presentan en media, desviación estándar, mínimo y máximo.

Se utilizó análisis bivariado: para determinar si existían diferencias entre la $\mathrm{SpO}_{2}$ y $\mathrm{FC}$, según vía de parto y género del recién nacido y se utilizó el Test $\mathrm{T}$ de Student o Mann Whitney, según distribución de los datos y $\chi^{2}$. El nivel de significancia utilizado fue de 0,05 .

\section{Resultados}

Al comparar la edad gestacional, Apgar al $1^{\text {er }}$ y $5^{\circ}$ minuto y género del RN, no se encontraron diferencias estadísticamente significativas según la vía de parto. Solo se encontraron diferencias significativas con respecto al peso de nacimiento, observándose un peso promedio significativamente menor en los $\mathrm{RN}$ por vía vaginal (tabla 1).

\section{Medición de $\mathrm{SpO}_{2}$ y frecuencia cardiaca}

El promedio y desviación estándar de la $\mathrm{SpO}_{2}$ del grupo analizado fue $58,7 \% \pm 11,6 \%$ (rango: $29-88 \%$ ), para el primer minuto, aumentando gradualmente a $83 \%$ a los $5 \min (46$ a $100 \%$ ) y posteriormente sobre $90 \%$ a los 8 min postparto (60 a 100\%). Respecto a la FC del grupo en estudio, al minuto el promedio y desviación estándar fue de $150 \pm 24,5$ lpm (rango:76-199 $\mathrm{lpm}$ ), alcanzando su mayor valor promedio a los $3 \mathrm{~min}$ $165 \mathrm{lpm}$ (75-198 lpm), para luego descender gradualmente llegando a $143 \mathrm{lpm}$ en promedio a los $60 \mathrm{~min}$ (95- $189 \mathrm{lpm}$ ) (tabla 2).

Al comparar la $\mathrm{SpO}_{2}$ según la vía de parto, se encontraron diferencias significativas hasta el minuto 10 postparto, observándose valores promedios significativamente mayores $(\mathrm{p}<0,05)$ en los partos vaginales, en comparación con los partos vía cesárea. Posteriormente, para los minutos 15,30 y 60 no se encontraron diferencias significativas (tabla 3 ).

Con respecto a la FC, se encontraron diferencias estadísticamente significativas en los primeros dos 
Tabla 1. Características demográficas de los 324 recién nacidos

\begin{tabular}{lccc}
\hline & Parto cesárea $(n=164)$ & Parto vaginal $(n=160)$ & Valor $p$ \\
\hline Edad gestacional, sem (media, rango) & $38,6(37-40)$ & $38,7(37-41)$ & 0,41 \\
Peso nacimiento, gramos (media, rango) & $3624(2230-4930)$ & $3415(2460-4330)$ & 0,0001 \\
Apgar 1 minuto (mediana, rango) & $9(8-9)$ & $9(7-10)$ & 0,80 \\
Apgar 5 min (mediana, rango) & $9(9-10)$ & $9(8-10)$ & 0,90 \\
Género Femenino $n, \%$ & $64(63,4)$ & $37(36,6)$ & 0,85 \\
Género Masculino $n, \%$ & $64(64,6)$ & $35(35,4)$ & \\
\hline
\end{tabular}

minutos postparto, observándose valores promedio mayores en los partos vía vaginal, en comparación a los partos vía cesárea, ocurriendo el mismo comportamiento en los minutos 10, 15, 30 y 60 , los cuales fueron significativamente mayores en los $\mathrm{RN}$ por vía vaginal (tabla 3).

$\mathrm{Al}$ comparar la $\mathrm{SpO}_{2}$ y FC según género del $\mathrm{RN}$, no se encontraron diferencias estadísticamente significativas, en ninguno de los minutos evaluados en este estudio (tabla 4).

En la figura 1, se observa que la saturación de oxígeno va aumentando gradualmente minuto a minuto hasta llegar a un valor superior al $90 \%$ al minuto 8 . Existen algunos neonatos que aún mantienen una $\mathrm{SpO}_{2}$ bajo $60 \%$ a los 5 y 6 min postparto, y 2 RN que presentaron una $\mathrm{SpO}_{2}$ menor o igual a $80 \%$ a los 10 min postparto. Con respecto a la FC, se observa neonatos con valores bajo $100 \mathrm{lpm}$, hasta los $7 \mathrm{~min}$ postparto. Sin embargo, desde el minuto 8 hasta los 60 min posparto, la FC es igual o superior a $100 \mathrm{lpm}$.
Tabla 2. $\mathrm{SpO}_{2}$ y FC durante los primeros 60 min post-natal en 324 recién nacidos

\begin{tabular}{ccc}
\hline $\begin{array}{c}\text { Tiempo } \\
\text { (min) }\end{array}$ & $\begin{array}{c}\mathrm{SpO}_{2}(\%) \\
\text { Media (rango) }\end{array}$ & $\begin{array}{c}\text { F.C. }(\mathrm{lpm}) \\
\text { Media }(\text { rango })\end{array}$ \\
\hline 1 & $58,7(29-88)$ & $150(76-199)$ \\
2 & $64,6(32-90)$ & $159(75-198)$ \\
3 & $71,3(36-96)$ & $165(75-198)$ \\
4 & $77,8(38-99)$ & $163(82-204)$ \\
5 & $83,0(46-100)$ & $163(96-200)$ \\
6 & $86,9(46-100)$ & $162(98-198)$ \\
7 & $89,5(64-100)$ & $160(98-194)$ \\
8 & $91,4(60-100)$ & $158(100-194)$ \\
9 & $92,8(64-100)$ & $157(100-200)$ \\
10 & $94,5(78-100)$ & $156(115-192)$ \\
15 & $96,1(86-100)$ & $152(120-193)$ \\
30 & $97,7(90-100)$ & $147(125-190)$ \\
60 & $98,3(89-100)$ & $143(125-189)$ \\
\hline
\end{tabular}

Ipm: latidos por minuto. $\mathrm{SpO}_{2}$ : saturación arterial de oxígeno. $\mathrm{FC}$ : frecuencia cardiaca.

Tabla 3. $\mathrm{SpO}_{2}$ y $\mathrm{FC}$ durante los primeros 60 min post-natal según vía de parto

\begin{tabular}{|c|c|c|c|c|c|c|}
\hline \multirow{2}{*}{$\begin{array}{l}\text { Tiempo } \\
\text { (min) }\end{array}$} & \multicolumn{2}{|c|}{ Saturación arterial de oxígeno (\%) } & \multirow[t]{2}{*}{ Valor $p$} & \multicolumn{2}{|c|}{ Frecuencia cardiaca (Ipm) } & \multirow[t]{2}{*}{ Valor $p$} \\
\hline & $\begin{array}{l}\text { Parto vaginal } \\
\text { Media (rango) }\end{array}$ & $\begin{array}{l}\text { Parto cesárea } \\
\text { Media (rango) }\end{array}$ & & $\begin{array}{l}\text { Parto vaginal } \\
\text { Media (rango) }\end{array}$ & $\begin{array}{l}\text { Parto cesárea } \\
\text { Media (rango) }\end{array}$ & \\
\hline 1 & $62,4 \quad(29-88)$ & $54,9 \quad(32-80)$ & $<0,0001$ & 155 (86-199) & $144(76-190)$ & $<0,01$ \\
\hline 2 & $69,0 \quad(32-90)$ & $60,0 \quad(32-87)$ & $<0,0001$ & 162 (90-198) & $156(75-198)$ & 0,01 \\
\hline 3 & $75,5 \quad(40-96)$ & $67,1 \quad(36-90)$ & $<0,0001$ & 166 (94-198) & $164(75-197)$ & 0,26 \\
\hline 4 & $81,6 \quad(50-99)$ & $74,1 \quad(38-95)$ & $<0,0001$ & 164 (98-204) & 163 (82-198) & 0,47 \\
\hline 5 & $86,0(58-100)$ & $80,0 \quad(46-96)$ & $<0,0001$ & 164 (99-200) & $162(96-195)$ & 0,23 \\
\hline 6 & $88,8(57-100)$ & $85,0 \quad(46-99)$ & $<0,0001$ & $163(112-198)$ & $160 \quad(98-196)$ & 0,28 \\
\hline 7 & $91,0(64-100)$ & $88,0(65-100)$ & $<0,0001$ & $161(118-194)$ & 159 (98-194) & 0,7 \\
\hline 8 & $92,2(60-100)$ & $90,6(64-100)$ & 0,02 & $160(117-194)$ & $157(100-191)$ & 0,63 \\
\hline 9 & $93,6(76-100)$ & $92,0(64-100)$ & 0,02 & $159(127-190)$ & $156(100-200)$ & 0,25 \\
\hline 10 & $95,1(80-100)$ & $93,9(78-100)$ & 0,01 & $158(126-190)$ & $154(115-192)$ & 0,02 \\
\hline 15 & $96,2(86-100)$ & $96,0(86-100)$ & 0,63 & $154(120-193)$ & $150(120-189)$ & 0,02 \\
\hline 30 & $97,7(90-100)$ & $97,5(90-100)$ & 0,26 & 149 (95-190) & $145(110-184)$ & $<0,01$ \\
\hline 60 & $98,3(89-100)$ & $98,2(92-100)$ & 0,62 & $143 \quad(95-170)$ & $142(115-189)$ & 0,04 \\
\hline
\end{tabular}

$\mathrm{SpO}_{2}$ : saturación arterial de oxígeno. FC: frecuencia cardiaca. Ipm: latidos por minuto. 
Tabla 4. $\mathrm{SpO}_{2}$ y $\mathrm{FC}$ durante los primeros 60 min post-natal según género del recién nacido

\begin{tabular}{|c|c|c|c|c|c|c|}
\hline \multirow{2}{*}{$\begin{array}{c}\text { Tiempo } \\
\text { (min) }\end{array}$} & \multicolumn{2}{|c|}{ Saturación arterial de oxígeno (\%) } & \multirow[t]{2}{*}{ Valor $\mathrm{p}$} & \multicolumn{2}{|c|}{ Frecuencia cardiaca (lpm) } & \multirow[t]{2}{*}{ Valor $p$} \\
\hline & $\begin{array}{c}\text { Femenino } \\
\text { Media (rango) }\end{array}$ & $\begin{array}{c}\text { Masculino } \\
\text { Media (rango) }\end{array}$ & & $\begin{array}{c}\text { Femenino } \\
\text { Media (rango) }\end{array}$ & $\begin{array}{c}\text { Masculino } \\
\text { Media (rango) }\end{array}$ & \\
\hline 1 & $57,23 \quad(29-80)$ & $58,53 \quad(36-88)$ & 0,44 & $144,99 \quad(76-188)$ & $147,59 \quad(77-189)$ & 0,43 \\
\hline 2 & $63,43 \quad(32-90)$ & $63,69 \quad(38-89)$ & 0,94 & $157,87 \quad(98-194)$ & $156,91 \quad(75-190)$ & 0,86 \\
\hline 3 & $70,79 \quad(47-95)$ & $70,52 \quad(40-95)$ & 0,98 & 165,97 (104-195) & $162,54 \quad(75-194)$ & 0,19 \\
\hline 4 & $77,49 \quad(48-96)$ & $77,79 \quad(56-99)$ & 0,91 & $163,92(108-194)$ & $161,13 \quad(82-196)$ & 0,25 \\
\hline 5 & $82,9 \quad(51-98)$ & $82,7 \quad(59-100)$ & 0,74 & $163,68(111-191)$ & $160,86 \quad(96-199)$ & 0,25 \\
\hline 6 & $86,91 \quad(67-98)$ & $87,22(68-100)$ & 0,74 & $163,1 \quad(122-193)$ & $158,74 \quad(98-196)$ & 0,08 \\
\hline 7 & $89,37(65-100)$ & $89,72(68-100)$ & 0,91 & $161,68(118-191)$ & 157,29 (98-194) & 0,07 \\
\hline 8 & $91,39(68-100)$ & $91,85(69-100)$ & 0,73 & $159,41(117-191)$ & $157,2 \quad(100-194)$ & 0,35 \\
\hline 9 & $93,01(72-100)$ & $92,77(64-100)$ & 0,77 & $157,76(123-189)$ & $155,51(100-192)$ & 0,32 \\
\hline 10 & $94,47(78-100)$ & $94,45(78-100)$ & 0,59 & $157,28(126-190)$ & $153,74(115-190)$ & 0,10 \\
\hline 15 & $96,42(89-100)$ & $96 \quad(86-100)$ & 0,25 & $152,91(120-193)$ & $150,81(120-185)$ & 0,29 \\
\hline 30 & $97,5 \quad(90-100)$ & $97,63(90-100)$ & 0,61 & $147,51(110-184)$ & 145,71 (120-190) & 0,33 \\
\hline 60 & $98,3 \quad(89-100)$ & $98,22(93-100)$ & 0,77 & $144,45(110-189)$ & $141,8 \quad(118-170)$ & 0,13 \\
\hline
\end{tabular}

$\mathrm{SpO}_{2}$ : saturación arterial de oxígeno. $\mathrm{FC}$ : frecuencia cardiaca. Ipm: latidos por minuto.

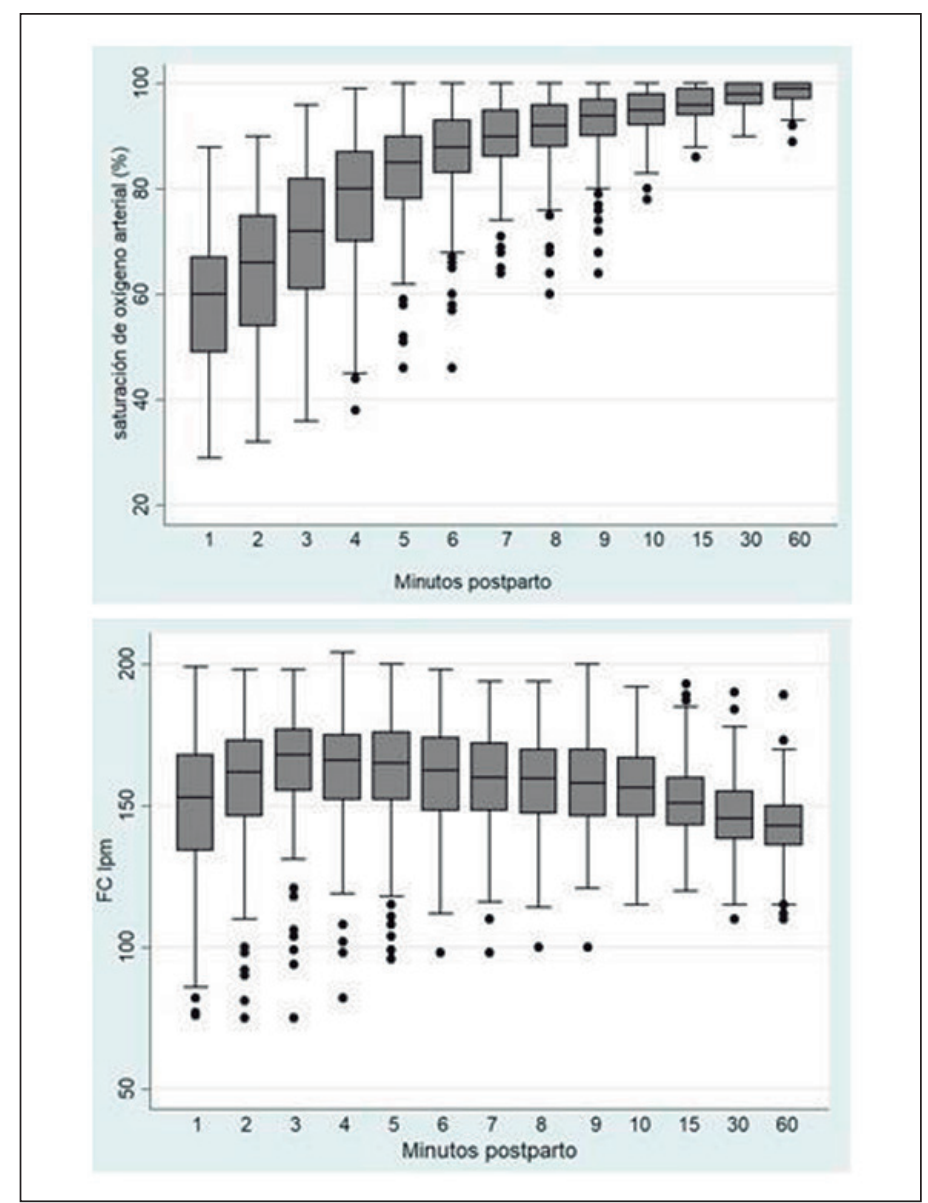

Figura 1. $\mathrm{SpO}_{2}$ y FC durante los primeros 60 min postparto en $324 \mathrm{RN}$. Las cajas representan los percentiles 25 y 75 y la línea dentro de esta representa la mediana. Las líneas superiores e inferiores fuera de las cajas corresponden a la dispersión, obtenidas mediante el rango intercuartílico. Los círculos representan los puntos atípicos (outliers)

\section{Discusión}

En la evaluación del RN la monitorización continua de la oxigenación y la frecuencia cardiaca, permiten evaluar el bienestar neonatal, cuya determinación es especialmente útil en los primeros minutos después del nacimiento ${ }^{2,4}$.

La $\mathrm{SpO}_{2}$ y la $\mathrm{FC}$ se midió en todos los neonatos desde el nacimiento, tanto en aquellos nacidos vía vaginal como por cesárea, que cumplieron con los criterios de inclusión; registros que se realizaron hasta los primeros 60 min post nacimiento. Los resultados del presente estudio confirman, al igual que otros autores ${ }^{7,8,15-18}$, que la oxigenación post-natal requiere de un período de adaptación de algunos minutos luego del parto, para lograr niveles de oxigenación dentro del rango normal. La baja saturación post-natal inicial se debería a la persistencia del corto circuito de derecha a izquierda por el ductus arterioso y por el foramen oval permeable. El ascenso de la $\mathrm{SpO}_{2}$ luego de 15 a 30 min a valores definitivos, corresponderían al cierre funcional de los corto circuitos a nivel ductal y atrial ${ }^{8,16}$.

En nuestro estudio la $\mathrm{SpO}_{2}$ mostró un ascenso progresivo desde $58,7 \%$ el primer minuto post nacimiento para alcanzar un $83 \%$ a los 5 min y sobrepasar el umbral del $90 \%$ a los 8 min y posteriormente sobre $95 \%$ a los 15 min post-natales; observación similar a lo reportado por otros autores ${ }^{7,8,15,16}$. Cabe destacar que el grupo de neonatos estudiados presentó una $\mathrm{SpO}_{2}$ promedio inferior a $80 \%$ en los primeros 4 min de vida post-natal; habiéndose demostrado que una saturación inferior a $80 \%$, puede asociarse con cianosis, debido a hipoxemia $\left(\mathrm{PaO}_{2}<50 \mathrm{mmHg}\right)$ y a los cambios que ocurren en la 
curva de disociación de la hemoglobina ${ }^{3,19}$. En consecuencia, es fisiológico esperar algún grado de cianosis en los primeros minutos post-natales del $\mathrm{RN}^{20}$.

Se ha demostrado también una gran variabilidad en la visualización de la cianosis en neonatos, junto con una pobre correlación entre ésta y la $\mathrm{SpO}_{2}{ }^{20,21}$. Según consenso de la Academia Americana de Pediatría y la Academia Americana del Corazón ${ }^{3}$, los RN que están respirando en forma espontánea, con FC normal, y buen tono muscular en los primeros minutos post-natales, si presentan cianosis, la suplementación con oxígeno es innecesaria; especialmente por los potenciales efectos tóxicos a mediano y largo plazo secundarios a la hiperoxigenación ${ }^{22}$.

La presente investigación al igual que en los estudios de Kamlin et $\mathrm{al}^{7}$, Harris et $\mathrm{al}^{9}$, Rabi et $\mathrm{al}^{15}$, Dawson et $\mathrm{al}^{23}$, Zubarioglu et $\mathrm{al}^{24} \mathrm{y}$ Zanardo et $\mathrm{al}^{25}$ los $\mathrm{RN}$ por cesárea presentaron una $\mathrm{SpO}_{2}$ significativamente menor que los nacidos vía vaginal en los primeros minutos post nacimiento. La menor $\mathrm{SpO}_{2}$ en los nacidos por cesárea, se debería al aumento de líquido pulmonar por falta de compresión del tórax fetal en el canal del parto, generando una alteración de la ventilaciónperfusión y por ende menor oxigenación? ${ }^{9}$ Según este estudio y de otros autores ${ }^{9,15}$, la reabsorción total o parcial del fluido pulmonar es un mecanismo rápido y transitorio, dado que la $\mathrm{SpO}_{2}$ por lo general se iguala a los $10 \mathrm{~min}$ en los neonatos nacidos por vía vaginal o cesárea. En los estudios de Dawson et $\mathrm{al}^{23}$, Harris et a ${ }^{9}$, y Rabi et $\mathrm{al}^{15}$ los valores promedio de $\mathrm{SpO}_{2}$ en los $\mathrm{RN}$ por vía vaginal o cesárea se igualaron a los 6,7 y a los 8 min post-natales, respectivamente.

En el estudio de Kamlin et $\mathrm{al}^{7}$ se desconoce en qué momento se igualan los valores de $\mathrm{SpO}_{2}$ entre los $\mathrm{RN}$ vía vaginal o cesárea, puesto que los valores de saturación sólo se registraron hasta los 5 min post nacimiento, tiempo en que la $\mathrm{SpO}_{2}$ fue significativamente mayor en los neonatos por vía vaginal.

En nuestro estudio, al igual que lo descrito por otros autores ${ }^{15,23}$, la $\mathrm{SpO}_{2}$ promedio fue superior a $90 \%$ a los 8 min post-natal. La diferencia entre nuestra experiencia y la de Rabi et a ${ }^{15}$ es que en esta última la $\mathrm{SpO}_{2}$ se midió preductal y a 1.000 metros sobre el nivel del mar (Presión atmosférica $660 \mathrm{mmHg}$ ) y la medición de nuestro estudio fue post-ductal y a nivel del mar. El estudio de Mariani y cols ${ }^{17}$, reveló un valor de $\mathrm{SpO}_{2}$ pre-ductal de $90 \%$ a los 5 min y un valor postductal superior a $90 \%$ a los 10 min post nacimiento. Sin embargo, otros autores han reportado mayores tiempos para lograr una $\mathrm{SpO}_{2}$ promedio post-ductal sobre $90 \%{ }^{8}$.

Los oxímetros de pulso junto con evaluar constantemente la $\mathrm{SpO}_{2}$ miden también simultáneamente la frecuencia cardiaca. Sin embargo, diversas publicaciones ${ }^{7-9,15-17}$, que evaluaron la $\mathrm{SpO}_{2}$ desde el nacimiento, no reportaron la FC, a pesar de ser considerada uno de los signos vitales más confiables, precisos y útiles para determinar la vitalidad del recién nacido y evaluar la respuesta a las maniobras de reanimación en caso de ser necesario ${ }^{26}$. Cabe destacar la importancia de la medición de la FC a través del oxímetro de pulso, puesto que se ha demostrado que la medición de FC a través de parámetros clínicos, como la auscultación o palpación, puede estar subestimada hasta en $17 \mathrm{lpm}^{26}$.

La FC promedio en nuestra casuística fue de 150 $1 \mathrm{pm}$ al primer minuto post-natal con un rango entre 76 y $199 \mathrm{lpm}$, la cual aumentó a $159 \mathrm{lpm}$ a los dos minutos post-natal, semejante a lo reportado por Toth y cols ${ }^{8}$ de $157 \mathrm{lpm}$ a los $2 \mathrm{~min}$ y por Meier- Stauss y $\mathrm{cols}^{27} \mathrm{de}$ $155 \mathrm{lpm}$ a los $3 \mathrm{~min}$ post-natales. El estudio realizado por Brady y $\mathrm{cols}^{28}$ referente a frecuencia cardiaca, hace más de 50 años, mostró una FC de 152 lpm al minuto post nacimiento, con un descenso de ésta a $140 \mathrm{lpm}$ a los 60 min post-natal, observación concordante con nuestra experiencia, que arrojó una FC de 143 lpm a los $60 \mathrm{~min}$ post nacimiento en el grupo estudiado. Sin embargo, estos hallazgos difieren del estudio de Dawson y $\operatorname{cols}^{23}$ en una población de $468 \mathrm{RN}$ de pretérmino y término, con una edad gestacional entre 25 y 42 semanas que mostró una $\mathrm{FC}$ promedio de $97 \mathrm{lpm}$ al primer minuto post-natal, de los cuales el $50 \%$ fueron menores a $100 \mathrm{lpm}$, aumentando a $139 \mathrm{lpm}$ al segundo minuto post-natal; frecuencia cardiaca inferior a la reportada por Toth y $\operatorname{cols}^{8}$ y a lo observado en el presente estudio. Los valores de FC de nuestro estudio, son concordantes con los de Toth y cols ${ }^{8}$ y Meier- Stauss ${ }^{27}$ los cuales reportaron aumento de la FC en los primeros 4 min post nacimiento con valores de $168 \mathrm{lpm}$ y $160 \mathrm{lpm}$ respectivamente, para luego estabilizarse a los $10 \mathrm{~min}$. Cabe hacer notar que en el estudio de Dawson $\mathrm{y} \operatorname{cols}^{23}$, la FC en los RN de pretérmino, fue inferior que en los de término, mostrando estos últimos, valores de FC semejantes a los nuestros desde el tercer minuto post-natal. Sin embargo, en el mismo estudio, la FC en los RN por vía vaginal fue significativamente mayor que los nacidos por cesárea, semejante a nuestra observación, en que los $\mathrm{RN}$ por vía vaginal, presentaron una FC mayor en los 2 primeros minutos post-natales y posteriormente desde el minuto $10 \mathrm{al} 60$, respecto a los RN por cesárea. Compartimos además otras similitudes respecto a lo reportado por Dawson y $\mathrm{cols}^{23}$, puesto que también registramos algunos $\mathrm{RN}$ con $\mathrm{FC}$ menor a $100 \mathrm{lpm}$ al primer minuto post nacimiento, la cual se recuperó rápidamente en forma espontánea, sin mediar acción médica. En el presente estudio, el reporte de FC menor a $100 \mathrm{lpm}$ en RN sanos al primer minuto post-natal, tal como fue reportado en un porcentaje significativo de neonatos por Dawson y $\operatorname{cols}^{23}$, es una observación interesante y que amerita mayores estudios; puesto que, según el algoritmo de reanimación de 
la $\mathrm{AAP}-\mathrm{AHA}^{3}$ al presentar el $\mathrm{RN}$ una $\mathrm{FC}<100 \mathrm{lpm}$ al primer minuto post-natal, sería indicación de realizar ventilación a presión positiva, intervención que no fue necesaria ni en el trabajo de Dawson y $\operatorname{cols}^{23}$ ni en el nuestro. Cabe señalar, que en nuestra serie se clampeó el cordón umbilical mayoritariamente luego de la primera respiración del recién nacido, evitándose el reflejo depresor, lo cual podría haber contribuido a registrar una FC mayor en los primeros 3 min post-natales. En nuestra investigación, los valores más elevados de FC del grupo total, respecto a lo reportado por Dawson y $\operatorname{cols}^{23}$, podría deberse a que este último estudio incluyó: RN prematuros, utilización de anestésicos o al hecho, que el clampeo del cordón, tal vez fue realizado, previo a la primera respiración del RN (evento no documentado), situaciones que por sí solas o en conjunto pudieran haber deprimido la FC del RN en los primeros minutos post-parto. En nuestra casuística las madres que fueron sometidas a cesárea, solo recibieron anestesia regional.

La medición de la FC por el oxímetro de pulso es una medición segura y más precisa que la simple auscultación ${ }^{26}$, por lo cual es improbable suponer algún tipo de error en la medición reportada por Dawson y $\operatorname{cols}^{23}$. Cabe señalar que nuestros datos de $\mathrm{SpO}_{2}$ y FC se analizaron, solo si presentaban una onda pletismográfica adecuada y una señal numérica de calidad; por lo cual, es improbable que hayan sido alteradas por artefactos. Los RN del presente estudio no recibieron oxigeno adicional y/o algún soporte respiratorio durante los primeros $60 \mathrm{~min}$ post-natales.

Las mediciones de $\mathrm{SpO}_{2}$ y FC realizadas por el oxímetro de pulso utilizado (Nellcor OxiMax N-600), en nuestro estudio, presenta una confiabilidad muy similar a los equipos que cuentan con tecnología de extracción de señal (Masimo) ${ }^{29}$.

A pesar de que diversas patologías cardiorrespiratorias son más prevalentes en el género masculino ${ }^{30,31}$, la mayoría de los estudios que han evaluado $\mathrm{SpO}_{2}$ pre y post-ductal en los primeros minutos post-natal, no han reportado que ocurre con la $\mathrm{SpO}_{2}$ respecto al género del $\mathrm{RN}^{7-9,15-18}$, excepto lo publicado por el grupo de autores de Turquía ${ }^{24,32}$, que no demostraron diferencias de género en la saturación de oxígeno tanto en RN de término como de pretérmino. Esta observación concuerda con lo encontrado en el presente trabajo, donde no se demostraron diferencias significativas tanto para la $\mathrm{SpO}_{2}$, como para la FC.
A diferencia de estudios anteriores ${ }^{7,15,17}$ registramos mediciones de la $\mathrm{SpO}_{2}$ y de FC durante los primeros 60 min post nacimiento y no sólo en los primeros 10 min post-natal, como en la mayoría de los estudios $^{5,28,32-34} \mathrm{o}$ hasta que la saturación fue superior a $95 \%{ }^{15,27}$.

Una de las posibles limitaciones de nuestro estudio, es que se midió saturometría post-ductal, con la finalidad de favorecer el apego. Dentro de las fortalezas se puede señalar un tamaño muestral significativo, y el hecho de haber sido realizado en un solo centro, lo que permitió una adecuada estandarización del estudio.

El presente estudio al igual que otros pre$\operatorname{vios}^{7-9,15-17,28,32-34}$ mostró la factibilidad de utilizar el oxímetro de pulso inmediatamente después del nacimiento, con la finalidad de monitorear en forma rápida, oportuna y precisa la saturación arterial de oxígeno y la frecuencia cardiaca del neonato.

En conclusión, en RN de término sanos la $\mathrm{SpO}_{2}$ aumenta progresivamente durante los primeros $10 \mathrm{~min}$ post-natal, siendo esta mayor en los primeros $7 \mathrm{~min}$ en los niños nacidos por vía vaginal. En los $\mathrm{RN}$ por vía vaginal, se observó una FC mayor en los 2 primeros minutos post-natal y luego desde el minuto 10 al 60 .

\section{Responsabilidades Éticas}

Protección de personas y animales: Los autores declaran que los procedimientos seguidos se conformaron a las normas éticas del comité de experimentación humana responsable y de acuerdo con la Asociación Médica Mundial y la Declaración de Helsinki.

Confidencialidad de los datos: Los autores declaran que han seguido los protocolos de su centro de trabajo sobre la publicación de datos de pacientes.

Derecho a la privacidad y consentimiento informado: Los autores han obtenido el consentimiento informado de los pacientes y/o sujetos referidos en el artículo. Este documento obra en poder del autor de correspondencia.

\section{Conflicto de intereses}

Los autores declaran no tener conflicto de intereses. 


\section{Referencias}

1. East C, Colditz P, Begg L. Update on intrapartum pulse oximetry; Aust N Z J Obstet Gynaecol 2002;42(2)119.

2. Saugstad O. Oxygen saturations immediately after birth. J. Pediatr 2006;148:569-70.

3. Kattwinkel J, Perlmann JM, Aziz K, et al. Neonatal Resuscitation 2010 American Heart Association Guidelines for Cardiopulmonary Resuscitation and Emergency Cardiovascular Care. Circulation 2010;122:S909-19.

4. Hay WW, Brockway JM, Eyzaguirre M. Neonatal pulse oximetry accuracy and reliability. Pediatrics 1989;83:717-20.

5. Dawson JA, Davis PG, O'Donnell CF, Kamlin CO, Morley CJ. Pulse Oximetry for Monitoring Infants in the Delivery Room: a Review. Arch Dis Child Fetal Neonatal Ed. 2007;92:F4-F7.

6. Wyllie J. Applied Physiology of newborn. Resuscitation Current Paediatrics 2006;16:379-385.

7. Kamlin O, Colm P, O Donnell C, Davis PG, Morley CJ. Oxygen saturation in healthy infants immediately after birth. J Pediatr 2006;148:585-9.

8. Toth B, Becker A, Seelbach-Gobel B. Oxygen saturation in healthy newborn infants immediately after birth measured by pulse oximetry. Arch Gynecol Obstet 2002;266:105-7.

9. Harris A, Sendak M, Donham M. Changes in Arterial Oxygen Saturation immediately after birth in the human neonate. J Pediatr 1986;109:117-9.

10. Gonzales GF, Salirrosas A. Arterial oxygen saturation in healthy newborns delivered at term in Cerro de Pasco (4340 m) and Lima (150m). Reproductive Biology and Endocrinology 2005;3:46-52.

11. Bancalari A, Moscoso P, Araya N, Araneda H. Arterial oxygen saturation in healthy newborns at sea level and at altitude from birth to the first day of life. E-PAS 2013;4516:265.

12. Levesque BM, Pollack P, Griffin BE,
Nielsen HC. Pulse Oximetry: What's is normal in the Newborn Nursery. Pediatrics Pulmonol 2000;30:406-12.

13. O'Donnell CP, Kamlin CO, Davis PG, Morley CJ. Feasibility of a delay in obtaining pulse oximetry during neonatal resuscitation. J Pediatr 2005;147:698-9.

14. Gungor S, Kurt E, Teksoz E, et al. Oronasopharyngeal suction versus no suction in normal and term infants delivered by elective cesarean section: a prospective randomized controlled trial. Gynecol Obstet Invest 2006;61:9-14.

15. Rabi Y, Yee W, Yue S, Singhal N. Oxygen Saturation Trends Immediately After Birth. J Pediatr 2006;148:590-4.

16. IDimich I, Singh P, Adell A, et al. Evaluation of oxygen saturation monitoring by pulse oximetry in neonates in the delivery system. Can J. Anaesth. 1991;38:985-8.

17. Mariani G, Dik PB, Ezquer A, et al. Preductal and post-ductal $\mathrm{O} 2$ saturation in healthy term neonates after birth. J Pediatr 2007;150:418-21.

18. Beskardes A, Salihoglu O, Can Emrah, et al. Oxygen saturation of healthy term neonates during the first 30 minutes of life. Pediatrics International 2013;55:44-8.

19. Oski FA, Delivoria-Papadopoulos M. The shift to the left. Pediatrics 1971;48:853-6.

20. O'Donnell CP, Kamlin CO, Davis PG et al. Clinical assessment of infant colour at delivery. Arch Dis Child Fetal Neonatal 2007;92:F465-7.

21. Goldman HI, Maralit A, Sun S, et al. Neonatal cyanosis and arterial oxygen saturation J Pediatr 1973;82:319-24.

22. Saugstad OD. Oxidative stress in the newborn: a 30-years perspective. Biol Neonate 2005;88:228-36.

23. Dawson JA, Kamlin COF, Vento M, et al. Defining the reference range for oxygen saturation for infants after birth. Pediatrics 2010;125:1340-6.

24. Zubarioglu U, Uslu S, Can E, Bülbül A, Nuhoglu A. Oxygen saturation levels during the first minutes of life in healthy term neonates. Tohoku J. Exp. Med 2011;224:273-9.

25. Zanardo V, Dal Cengio V, Trevisanuto D, et al. Elective caesarean delivery adversely affects preductal oxygen saturation during birth transition; Arch Dis Child Fetal Neonatal Ed 2016;101:339-43.

26. Kamlin CO, Donnell C, Everest N, et al. Accuracy of clinical assessment of infant heart rate in the delivery room Resuscitation 2006;71:319-221.

27. Meier-Stauss $P$, Bucher HU, Hürlimann R, Körnig V, Huch R. Pulse oximetry used for documenting oxygen saturation and right-to left shunting immediately after birth. Eur J Pediatr 1990;149:851-5.

28. Brady JP, James LS. Heart rate changes in the fetus and newborn infant during labor delivery, and the immediate neonatal period. Am J Obstet Gynecol 1962;84:112.

29. Dawson J, Saraswat A, Davis PG, et al. Comparison of heart rate and oxygen saturation measurements from Masimo and Nellcor pulse oximeters in newly born term infants; Acta Pediatrica 2013;102:1 6.

30. Townsel C, Emmer S, Campbell W. Gender Differences in Respiratory Morbidity and Mortality of Preterm Neonates. Frontiers in Pediatrics 2017;5:6.

31. Peacock J, Marston L, Marlow N. Neonatal and infant outcome in boys and girls born very prematurely; Pediatric Research 2012;71(3):305-310.

32. Uslu S, Zubarioglu U, Sozeri S, et al. Factors Affecting the Target Oxygen Saturation in the First Minutes of Life in Preterm Infants; Journal of Tropical Pediatrics 2017;63:286-93.

33. Alcuntu E, OzeK E Bilgen $\mathrm{H}$, et al. Percentiles of oxygen saturations in healthy term newborns in the first minutes of life. Eur J Pediatr 2008;167:687-8.

34. House JT, Schultetus RR, Gravenstein N. Continuous neonatal evaluations in the delivery rooms by pulse oximetry. J Clin Monit 1987;3:96-100. 